\title{
Common Behaviors of Spinor-Type Instantons in 2D Thirring and 4D Gursey Fermionic Models
}

\author{
Fatma Aydogmus ${ }^{1}$ and Eren Tosyali ${ }^{2}$ \\ ${ }^{1}$ Department of Physics, Istanbul University, Istanbul, Turkey \\ ${ }^{2}$ School of Advanced Vocational Studies, Istanbul Bilgi University, Istanbul, Turkey \\ Correspondence should be addressed to Fatma Aydogmus; fatma.aydogmus@gmail.com
}

Received 1 March 2014; Accepted 17 June 2014; Published 17 July 2014

Academic Editor: Shi-Hai Dong

Copyright (c) 2014 F. Aydogmus and E. Tosyali. This is an open access article distributed under the Creative Commons Attribution License, which permits unrestricted use, distribution, and reproduction in any medium, provided the original work is properly cited. The publication of this article was funded by SCOAP ${ }^{3}$.

\begin{abstract}
We investigate two examples of conformal invariant pure spinor fermionic models, which admit particle-like solutions of the classical field equations. For different dimensions and quantum spinor numbers, the vector field visualizations of the models are constructed to provide a better understanding of the spinor-type instanton dynamics in phase space. The hierarchical cluster analysis method investigations of the models are also presented. Finally, the autocorrelation and power spectrum graphs of models are constructed and frequencies of motions are defined.
\end{abstract}

\section{Introduction}

The success of electron and antielectron (positron) interpretation of Dirac's nonlinear spinor field wave equation brought with itself a great effort among theoretical physicists in searching new nonlinear field equations. Heisenberg spent his years to formulate a "theory of everything" using only fermions [1]. A few years later another attempt in this direction came with the work of Gursey [2]. Gursey proposed his model as a possible basis for a unitary description of elementary particles to remedy some of the problems the Heisenberg model had. Gursey model is the first conformally invariant pure fermionic model and it is four-dimensional [2]. It possesses broader dynamical symmetries compared to Dirac's and Heisenberg et al.s works. What is more important is that Gursey's model is suitable for extensions to other particles having spin. In the ensuing years, Kortel found a class of exact solutions of Gursey spinor wave equation and these were shown to be instantonic and meronic in character [3, 4]. After the spinor-type instanton solutions of this model with nonlinear $(\bar{\psi} \psi)^{4 / 3}$ self-coupled spinor term have been found by the spontaneous symmetry breaking of the conformal invariance of $\psi$, that is, $\langle 0|\bar{\psi} \psi| 0\rangle \neq 0$ [4], many works have been done on this model [5-9]. Gursey model is very important because of the similarity of these solutions to the solutions of pure Yang-Mills theories in four dimensions.

Another fermionic model is the Thirring model which describes Dirac fermions in $(1+1)$ space-time dimensions with the nonlinear $(\bar{\psi} \psi)^{2}$ self-interaction term [10]. Thirring model is also conformally invariant and with no mass term. It played a very fruitful role in the progress of quantum field theory [10]. The spinor-type instanton solutions of Thirring model were also found via breaking of conformal symmetry and were shown that these solutions were stable [11]. This model is simple enough to be analyzed with full details; and yet it contains many of the typical features of the quantization of relativistic quantum field theories [12].

It is well known that instantons are classical topological solutions in quantum field theories which lie behind the standard model of particles. The aim of this study is to provide a better understanding to the dynamics of spinortype instanton solutions of fermionic models in phase space for different dimensions and quantum spinor numbers. For this purpose, the vector fields of conformal invariant pure spinor fermionic models are constructed and their Jacobian determinants are calculated. In addition, the autocorrelations and power spectra [13] are presented to see whether the oscillations are periodic or not. In the periodic oscillation case, the 
oscillation frequencies of motions are calculated. Moreover, hierarchical cluster analysis [14] method investigations of the fermionic models are presented.

\section{Models}

Below we present firstly the behavior of spinor-type instantons in Thirring model because of the lower space-time dimensionality and later do the same for Gursey model.

2.1. Thirring Model. In this model the two-dimensional fermion-fermion interactions are described by the conformal invariant Lagrangian

$$
L=i \bar{\psi} \sigma_{\mu} \partial_{\mu} \psi+\frac{g}{2}(\bar{\psi} \psi)^{2}
$$

where $g$ is the positive coupling constant and the fermion field $\psi(x)$ has scale dimension $1 / 2$ [10]. The equation of motion that follows is

$$
i \sigma_{\mu} \partial_{\mu} \psi+g(\bar{\psi} \psi) \psi=0
$$

The complex form of the Euclidian configuration of Heisenberg ansatz [1] is

$$
\psi=\left[i x_{\mu} \gamma_{\mu} \chi(s)+\varphi(s)\right] C,
$$

with an arbitrary spinor constant, C. $\chi(s)$, and $\varphi(s)$ are real functions of $s=x^{2}+t^{2}\left(x_{1} \equiv x, x_{2} \equiv t\right)$. Inserting (3) into (2) we get

$$
\begin{gathered}
\chi(s)+s \frac{d \chi(s)}{d s}+\alpha\left[s \chi(s)^{2}+\varphi(s)^{2}\right] \varphi(s)=0, \\
\frac{d \varphi(s)}{d s}-\alpha\left[s \chi(s)^{2}+\varphi(s)^{2}\right] \chi(s)=0,
\end{gathered}
$$

where $\alpha=g(\bar{C} C)$, since

$$
\begin{gathered}
i \not \partial \psi=i \sigma_{\mu} \partial_{\mu} \psi \\
=\left[-2 \chi(s)-2 s \frac{\mathrm{d} \chi(s)}{\mathrm{d} s}+2 i x_{\mu} \sigma_{\mu} \frac{\mathrm{d} \varphi(s)}{\mathrm{d} s}\right] \bar{C} C, \\
(\bar{\psi} \psi)=\left(s \chi(s)^{2}+\varphi(s)^{2}\right)(\bar{C} C) .
\end{gathered}
$$

By writing $\chi=A s^{-\sigma} F(u)$ and $\varphi=B s^{-\tau} G(u)$, with $u \equiv \ln s$ and $\sigma=\tau+1 / 2, \tau=1 / 4$, and $A^{2}=B^{2}$ [3], we achieved the dimensionless form of the nonlinear ordinary coupled differential equation system (4a) and (4b) as

$$
\begin{aligned}
& 2 \frac{d F(u)}{d u}+\frac{1}{2} F(u)-\alpha A B\left(F(u)^{2}+G(u)^{2}\right) G(u)=0, \\
& 2 \frac{d G(u)}{d u}-\frac{1}{2} G(u)+\alpha A B\left(F(u)^{2}+G(u)^{2}\right) F(u)=0 .
\end{aligned}
$$

Here $F$ and $G$ are dimensionless functions of $u$ and $A, B$ are constants [3]. We call the equation system in (6a) and (6b) as the Thirring nonlinear differential equation system (TNDES). The solution of TNDES for $\alpha(A B)=1$ is the Thirring instantons given in [11]. Recently, the role of the coupling constant in the evolution of Thirring instantons in phase space has been investigated $[8,15]$.
2.2. Gursey Model. In this 4-dimensional model the Lagrangian is based on fermion fields of scale dimension $3 / 2$ [2]

$$
L=i \bar{\psi} \not \partial+g(\bar{\psi} \psi)^{4 / 3}
$$

The equation of motion that follows is

$$
i \not \partial \psi+g(\bar{\psi} \psi)^{1 / 3} \psi=0 .
$$

The same ansatz (see (3)) is also used to solve (8) [3]. Here $s=x_{\mu}^{2}=r^{2}+t^{2}\left(x_{1} \equiv x, x_{2} \equiv y, x_{3} \equiv z ; x_{4} \equiv t\right)$. Inserting (3) into (8) we get

$$
\begin{gathered}
4 \chi(s)+2 s \frac{\mathrm{d} \chi(s)}{\mathrm{d} s}-\alpha\left[s \chi(s)^{2}+\varphi(s)^{2}\right]^{1 / 3} \varphi(s)=0, \\
2 \frac{\mathrm{d} \varphi(s)}{\mathrm{d} s}+\alpha\left[s \chi(s)^{2}+\varphi(s)^{2}\right]^{1 / 3} \chi(s)=0,
\end{gathered}
$$

where $\alpha=g(\bar{C} C)^{1 / 3}$ with

$$
\begin{gathered}
i \gamma_{\mu} \partial_{\mu} \psi=\left[-4 \chi(s)-2 s \frac{\mathrm{d} \chi(s)}{\mathrm{d} s}+2 i x_{\mu} \gamma_{\mu} \frac{\mathrm{d} \varphi(s)}{\mathrm{d} s}\right] \bar{C} C, \\
(\bar{\psi} \psi)^{1 / 3}=\left(s \chi^{2}(s)+\varphi^{2}(s)\right)(\bar{C} C)^{1 / 3}
\end{gathered}
$$

Substituting $\chi=A s^{-\sigma} F(u)$ and $\varphi=B s^{-\tau} G(u)$, with $u \equiv \ln s$, $\sigma=\tau+1 / 2, \tau=3 / 4$, and $A^{2}=B^{2}$ [3], we achieved the dimensionless form of the nonlinear ordinary coupled differential equation systems (9a) and (9b) as

$$
\begin{aligned}
& 2 \frac{\mathrm{d} F(u)}{\mathrm{d} u}+\frac{3}{2} F(u)-\alpha(A B)^{1 / 3}\left[F(u)^{2}+G(u)^{2}\right]^{1 / 3} G(u)=0 \\
& 2 \frac{\mathrm{d} G(u)}{\mathrm{d} u}-\frac{3}{2} G(u)+\alpha(A B)^{1 / 3}\left[F(u)^{2}+G(u)^{2}\right]^{1 / 3} F(u)=0 .
\end{aligned}
$$

We call this equation system the Gursey nonlinear differential equation system (GNDES). The solution of GNDES for $\alpha(A B)^{1 / 3}=1$ is the Gursey instantons given in [4]. Very recently, the role of the coupling constant in the evolution of Gursey instantons in phase space has been investigated $[8,9]$. For a better understanding of the quantum dynamics of spinor-type instantons in vacuum, the dynamics of Forced Gursey nonlinear differential equation system have been studied $[8,16]$.

\section{Vector Analysis of the Models}

TNDES can be written in the form of a vector field [17] as follows by defining a new constant $\beta \equiv \alpha(A B)$

$$
\mathbf{f}_{T}=\left(-\frac{1}{4} F+\frac{1}{2} \beta\left[F^{2}+G^{2}\right] G, \frac{1}{4} G-\frac{1}{2} \beta\left[F^{2}+G^{2}\right] F\right)
$$

since

$$
\nabla \cdot \mathbf{f}_{T}=\left(-\frac{1}{4}+F G \beta+\frac{1}{4}-F G \beta\right)=0
$$


TABLE 1: Characterization of the fix points.

\begin{tabular}{lccc}
\hline$\beta$ parameter & Fix points $\left(F^{*}, G^{*}\right)$ & Eigenvalues $\lambda_{ \pm}$ & Characterization \\
\hline 0 & $(0,0)$ & $\pm \frac{1}{4}$ & Hyperbolic \\
0.5 & $(0,0) ;\left( \pm \frac{1}{2 \sqrt{0.5}}, \pm \frac{1}{2 \sqrt{0.5}}\right)$ & $\pm \frac{1}{2} i$ & Elliptic \\
1 & $(0,0) ;\left( \pm \frac{1}{2}, \pm \frac{1}{2}\right)$ & $\pm \frac{1}{2} i$ & Elliptic \\
1.5 & $(0,0) ;\left( \pm \frac{1}{2 \sqrt{1.5}}, \pm \frac{1}{2 \sqrt{1.5}}\right)$ & $\pm \frac{1}{2} i$ & Elliptic \\
2 & $(0,0) ;\left( \pm \frac{1}{2 \sqrt{2}}, \pm \frac{1}{2 \sqrt{2}}\right)$ & $\pm \frac{1}{2} i$ & Elliptic \\
\hline
\end{tabular}

${ }^{*}$ refers to fix points that don't change upon application of differential equation system.

Due to the Liouville theorem TNDES is conserved, as is also seen from the vector field displays given in Figure 1.

On the other hand, the Jacobian of TNDES is

$$
\begin{gathered}
J_{T}=\left(\begin{array}{cc}
-\frac{1}{4}+F G \beta & -F^{2} \beta-\frac{1}{2}\left(F^{2}+G^{2}\right) \beta \\
G^{2} \beta+\frac{1}{2}\left(F^{2}+G^{2}\right) \beta & +\frac{1}{4}-F G \beta
\end{array}\right), \\
\operatorname{det}\left(J_{T}\right)=-\frac{1}{16}+\frac{F G \beta}{2}+\frac{3 F^{4} \beta^{2}}{4}+\frac{3}{2} F^{2} G^{2} \beta^{2}+\frac{3 G^{4} \beta^{2}}{4} .
\end{gathered}
$$

In Figure 1(a), the vector field of TNDES is displayed for $\beta=$ 0 . Figures $1(\mathrm{~b})-1(\mathrm{~d})$ show the vector field display of TNDES for $\beta=0.5,1$, and 1.5 , respectively. $\beta=1$ is the special case; instanton type solutions, called "Thirring instantons," have been found [11]. Figure $2 \operatorname{shows} \operatorname{det}\left(J_{T}\right)$ behavior versus $F$ and $G$ for $\beta=1$. $\operatorname{det} J_{T}$ is clearly positive outside of a tiny neighbor of $(0,0)$ in which it is negative; therefore, the flow is orientation preserving (Figure $1(\mathrm{c})$ ). The system is conservative along the flow.

The characteristic eigenvalues providing an understanding of the topological behavior around the singularity points come from $\left|J_{T}-\lambda I\right|=0$ as

$$
\lambda_{ \pm}= \pm \frac{1}{4} \sqrt{1-8 \beta F G-12 \beta^{2} F^{4}-12 \beta^{2} G^{4}-24 \beta^{2} F^{2} G^{2}} .
$$

Table 1 presents the fix points, their eigenvalues, and characterizations at three $\beta$ values in the $0 \leq \beta \leq 2$ interval. We replaced fix points into (15) to find the behavior of the system around the fix points. For $\beta=0$, one finds reel eigenvalues. Hence the fix point is hyperbolic, as is seen in Figure 1(a). For the other $\beta$ values, eigenvalues are purely imaginary. So the fix points are elliptic. These results are compatible with the phase space displays seen in Figure 1.

Analogously, GNDES can be written in the form of a vector field as follows, by defining a new constant $\gamma \equiv$ $\alpha(A B)^{1 / 3}$ :

$$
\mathbf{f}_{G}=\left(-\frac{3}{4} F+\frac{1}{2} \gamma\left[F^{2}+G^{2}\right]^{1 / 3} G, \frac{3}{4} G-\frac{1}{2} \gamma\left[F^{2}+G^{2}\right]^{1 / 3} F\right) .
$$

We take the divergence of $\mathbf{f}_{G}$

$$
\nabla \cdot \mathbf{f}_{G}=\left(-\frac{3}{4}+\frac{F G \gamma}{3\left(F^{2}+G^{2}\right)^{2 / 3}}+\frac{3}{4}-\frac{F G \gamma}{3\left(F^{2}+G^{2}\right)^{2 / 3}}\right)=0
$$

Density is conserved since $\nabla \cdot \mathbf{f}=0$; so phase space volumes are conserved as in Figure 3. We find the Jacobian of GNDES as
Alike, the vector fields of GNDES are shown in Figures 3(a)$3(\mathrm{~d})$ and the evolution of $\operatorname{det} J_{G}$ versus $F$ and $G$ is shown in
Figure 4 for $\gamma=1$. For $\gamma=1$, instanton type solutions called "Gursey instantons" have been found [4]. In comparison 


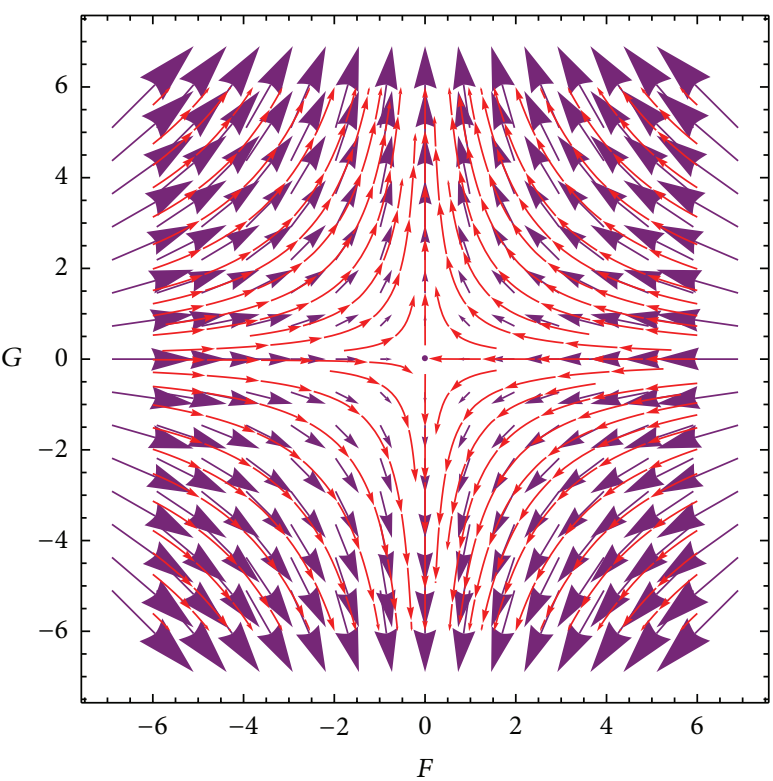

(a)

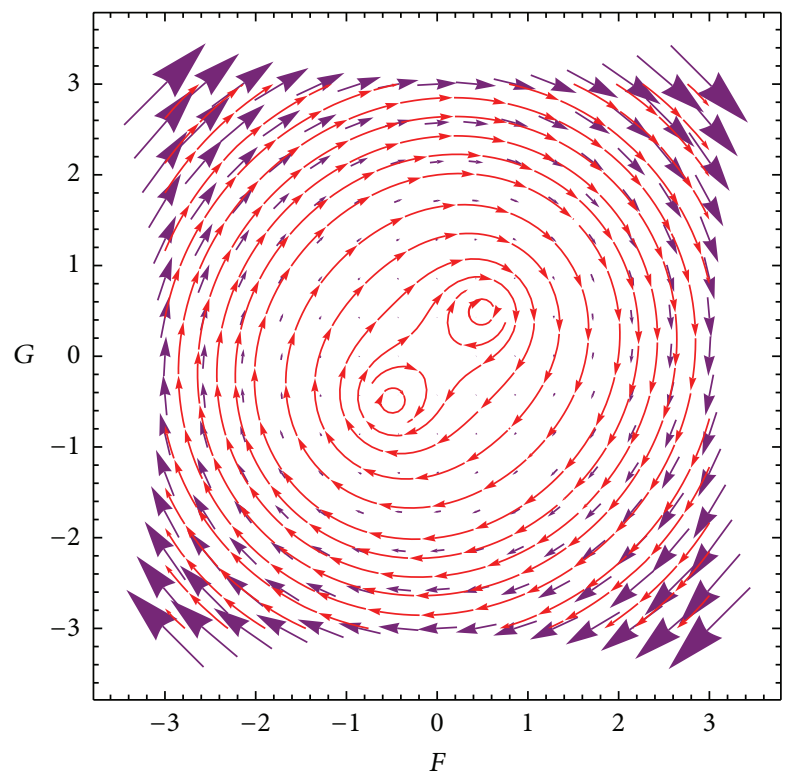

(c)

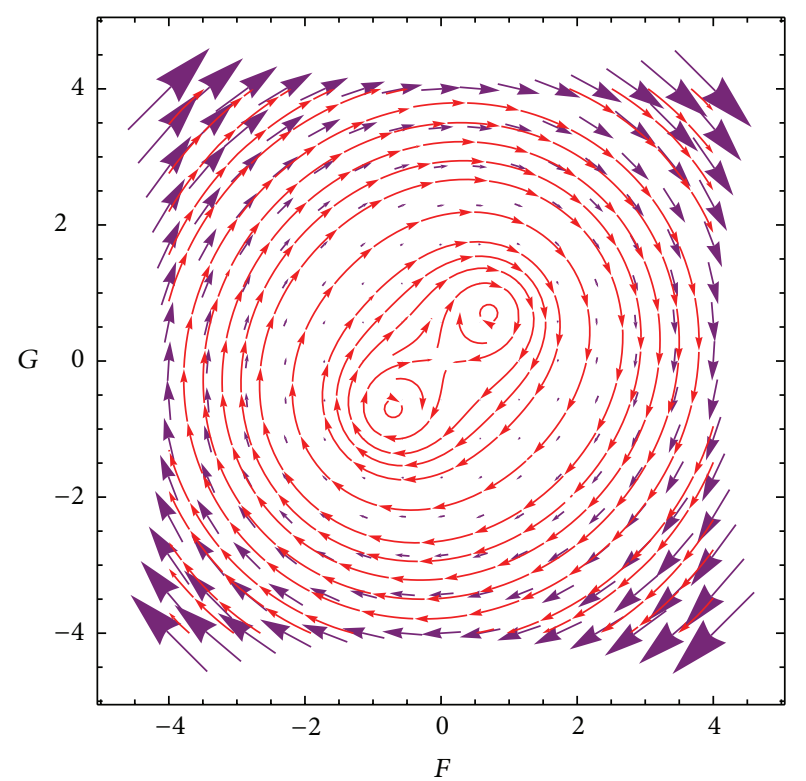

(b)

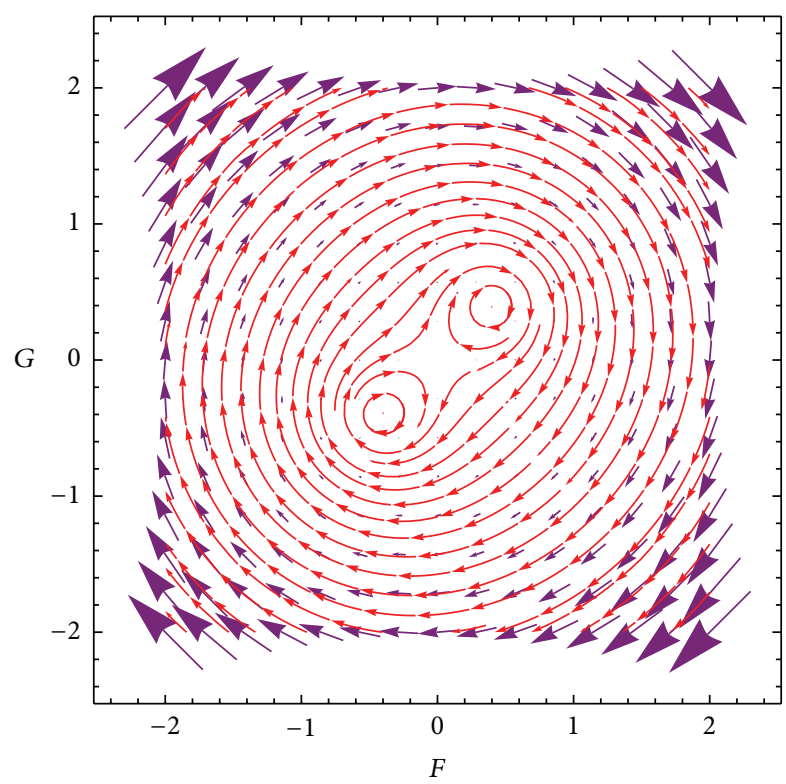

(d)

FIGURE 1: Vector field display of TNDES for (a) $\beta=0$, (b) $\beta=0.5$, (c) $\beta=1$, and (d) $\beta=1.5$.

with Figures 1(a) and 3(a), the vector visualization of Gursey instantons has the same behavior in phase space like Thirring instantons. The Gursey flow is also conservative.

In this case the characteristic eigenvalues come from $\mid J_{G^{-}}$ $\lambda I \mid=0$ as

$$
\begin{aligned}
& \lambda_{ \pm} \\
& = \pm\left(27-\frac{24 \gamma F G}{\left(F^{2}+G^{2}\right)^{2 / 3}}-\frac{8 \gamma^{2} F^{2}}{\left(F^{2}+G^{2}\right)^{1 / 3}}\right.
\end{aligned}
$$

$$
\begin{aligned}
& \left.-\frac{8 \gamma^{2} G^{2}}{\left(F^{2}+G^{2}\right)^{1 / 3}}-12 \gamma^{2}\left(F^{2}+G^{2}\right)^{2 / 3}\right)^{1 / 2} \\
& \times(4 \sqrt{3})^{-1} .
\end{aligned}
$$

Table 2 presents the fix points, their eigenvalues, and characterizations for five $\gamma$ values in the $0 \leq \gamma \leq 2$ interval. For $\gamma=0$, one finds reel eigenvalues. Hence the fix point is hyperbolic, as seen in Figure 3(a). For the other $\gamma$ values, eigenvalues are purely imaginary. So the fix points are elliptic 
TABLE 2: Characterization of the fix points.

\begin{tabular}{lccc}
\hline$\gamma$ parameter & Fix points $\left(F^{*}, G^{*}\right)$ & Eigenvalues $\lambda_{ \pm}$ & Characterization \\
\hline 0 & $(0,0)$ & $\pm \frac{3}{4}$ & Hyperbolic \\
0.5 & $(0,0) ;\left( \pm 3 \sqrt{\frac{3}{2}}, \pm 3 \sqrt{\frac{3}{2}}\right)$ & $\pm \frac{\sqrt{3}}{2} i$ & Elliptic \\
1 & $(0,0) ;\left( \pm \frac{3 \sqrt{3}}{4}, \pm \frac{3 \sqrt{3}}{4}\right)$ & $\pm \frac{\sqrt{3}}{2} i$ & Elliptic \\
1.5 & $(0,0) ;\left( \pm \frac{1}{\sqrt{2}}, \pm \frac{1}{\sqrt{2}}\right)$ & $\pm \frac{\sqrt{3}}{2} i$ & Elliptic \\
2 & $(0,0) ;\left( \pm \frac{3 \sqrt{3 / 2}}{8}, \pm \frac{3 \sqrt{3 / 2}}{8}\right)$ & $\pm \frac{1}{2}$ & Elliptic \\
\hline
\end{tabular}

${ }^{*}$ refers to fix points that don't change upon application of differential equation system.

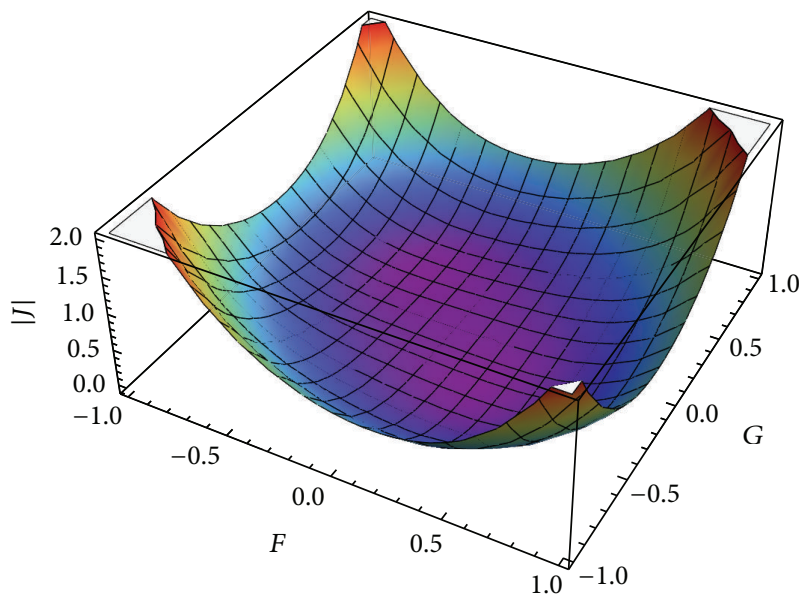

(a)

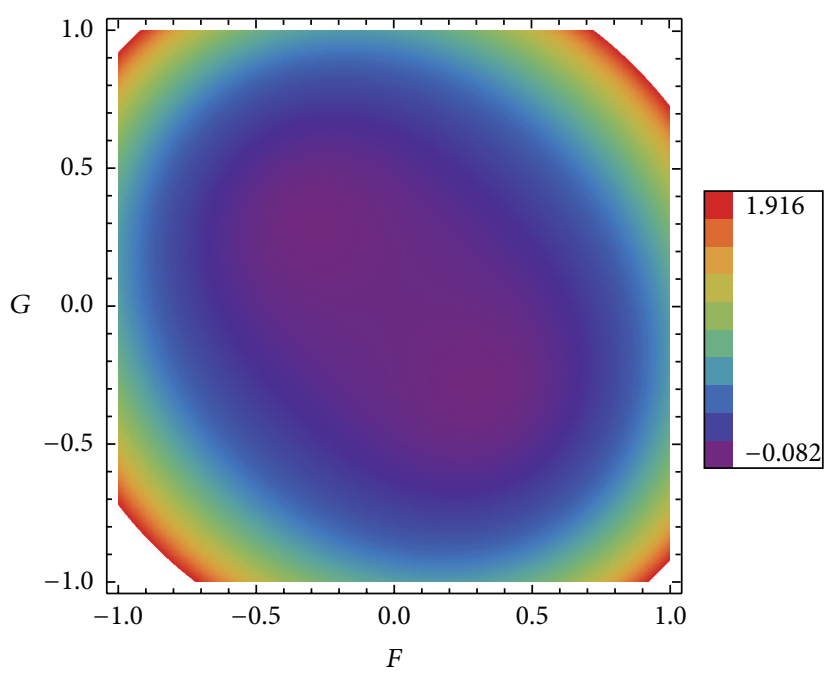

(b)

Figure 2: (a) 3D plot and (b) contour plot of $\operatorname{det}\left(J_{T}\right)$ in the $\beta=1$ case which accepts "Thirring instantons." as in the Thirring case. These results are compatible with the phase space displays seen in Figure 3.

\section{Cluster Analysis of the Models}

As additional information, we study the models using the hierarchical cluster analysis method. Hierarchical methods usually produce a graphical output known as a dendrogram graph that shows the hierarchical clustering structure [14]. In Figure 5, the dendrogram graphs belonging to hierarchical clustering of time series for TNDES solutions $(F(u))$ for $\beta=$ $0.5,1$, and 1.5 are shown. In these graphs, the $x$-axis represents the similarity or correlation percentages (\%0-\%100) belonging to our data $F(u)$ and increases towards right. Along the $y$-axis the fusion of clusters due to similarities of $F(u)$ data is recorded. For each $\beta$ value we plotted the dendrogram graphs for all data and give only the truncate dendrogram graphs which are the summary of the first 20 mergers having the same similarity percentage. From hierarchical point of view, for all $\beta$ values clustering is gathered in two different main groups. However, for $\beta=0.5$ there are five different clusterings in the first main cluster; in the second main cluster there are ten different clusterings and for $\beta=1$ there are six in the first main cluster. In the second main cluster there are twelve different clusterings. Finally, for $\beta=1.5$, there are seven different clusterings in the first main cluster and only twelve different clusterings in the second main one.

On the other hand, the dendrogram graphs belonging to the hierarchical clustering of time series for GNDES solutions $(F(u))$ for $\gamma=0.5,1$, and 1.5 are shown in Figure 6. Analogously for each $\gamma$ value we plotted the dendrogram graphs for all data values and give only the truncate dendrogram graphs which are the summary of the first 20 mergers having the same similarity percentage. For all $\gamma$ values, clustering is gathered in two different main groups comparably TNDES. However, for $\gamma=0.5$ there are four different clusterings in the first main cluster; in the second main cluster there are eleven different clusterings and for $\gamma=1$ in the first main cluster there are seven different clusterings and in the second main cluster there are fourteen different clusterings as distinct 


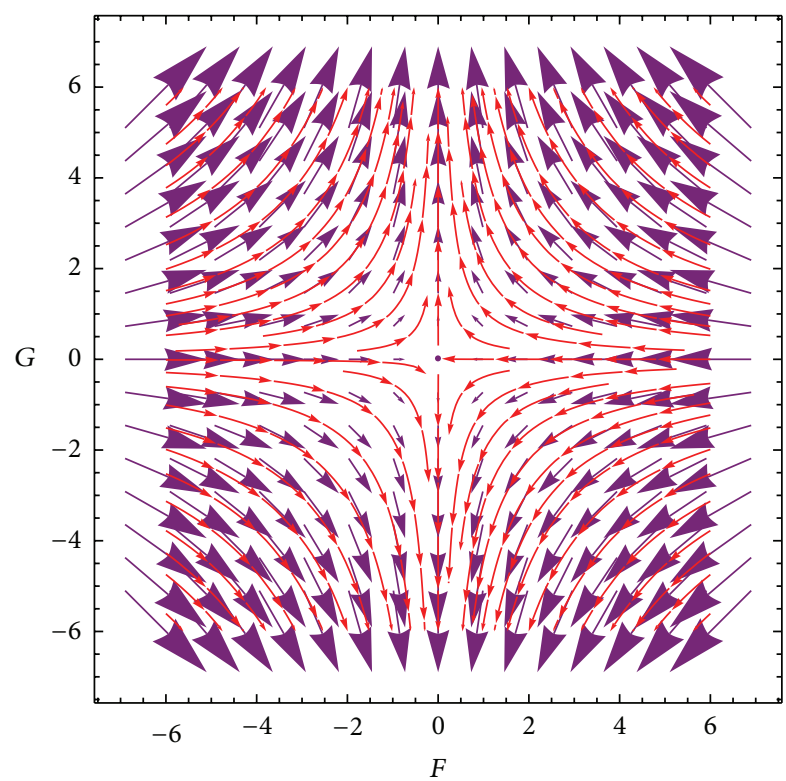

(a)

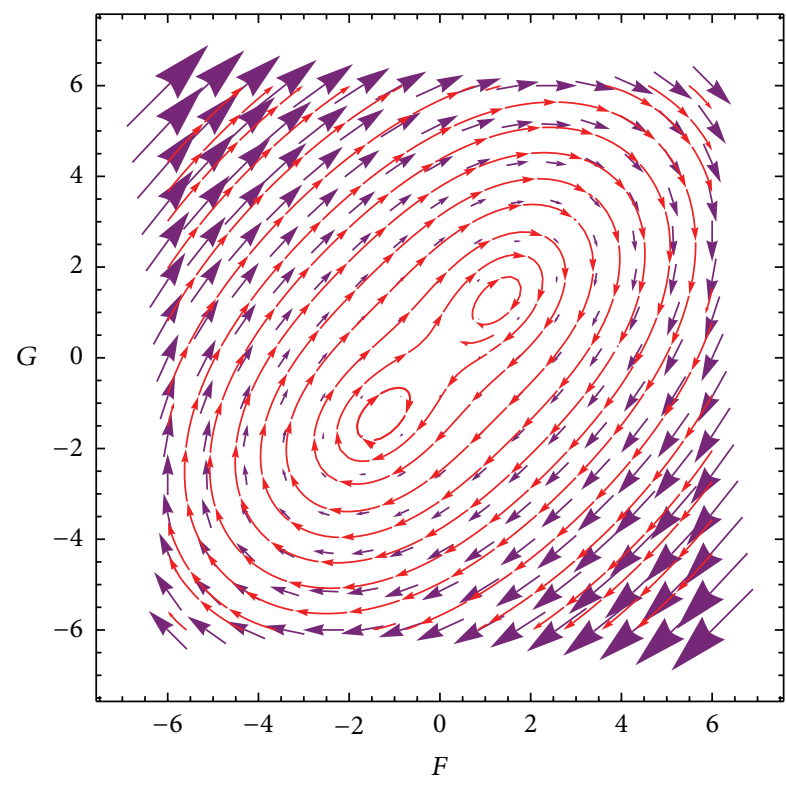

(c)

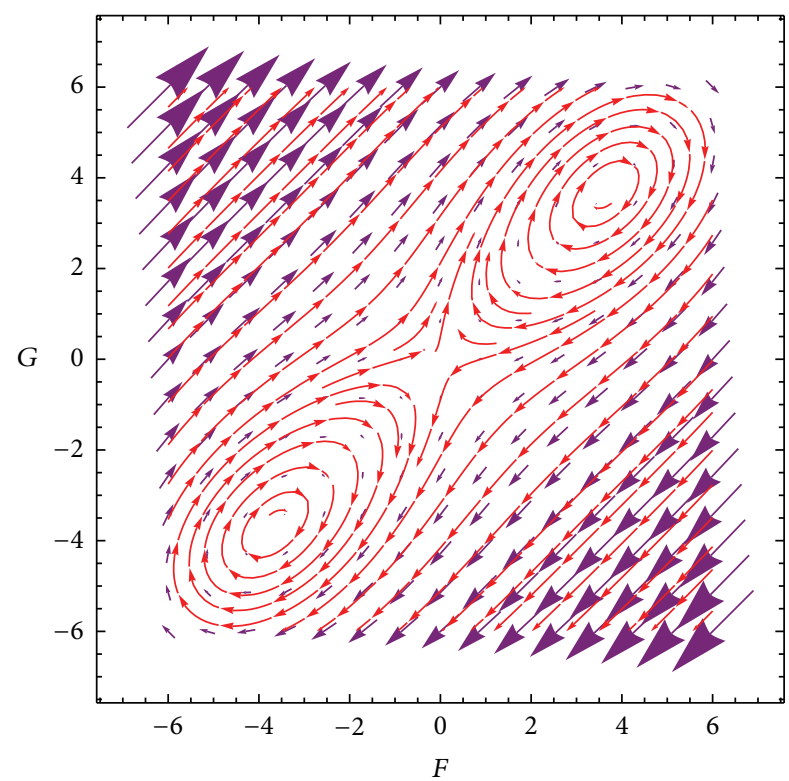

(b)

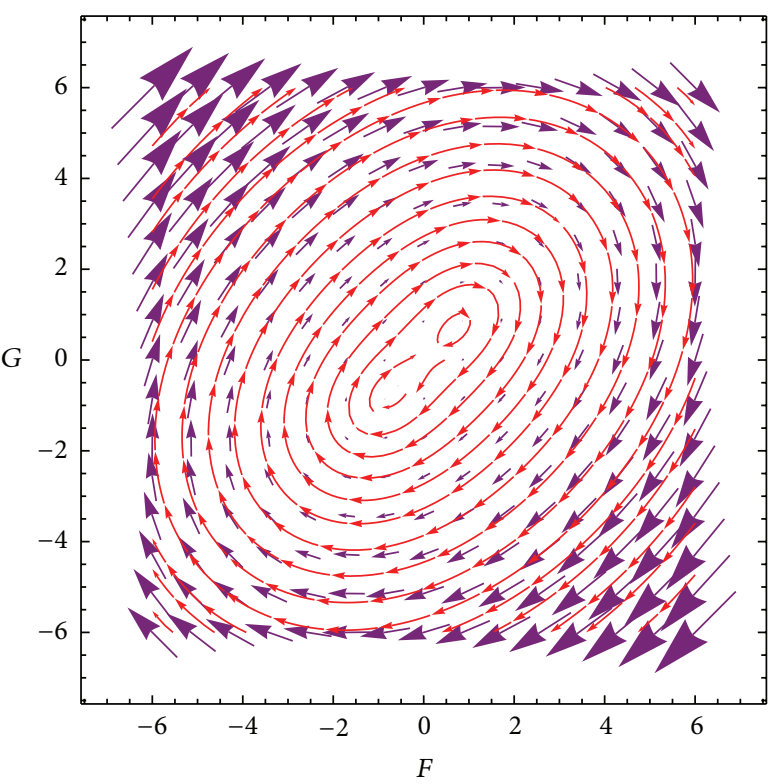

(d)

FIGURE 3: Vector field displays of GNDES for (a) $\gamma=0$; (b) $\gamma=0.5$; (c) $\gamma=1$; and (d) $\gamma=1.5$.

from TNDES. Besides for $\gamma=1.5$, there are seven different clusterings in the first main cluster and only thirteen different clusterings in the second main one.

\section{Autocorrelations and Power Spectrums of the Models}

Autocorrelation gives similarities at two points in a time series as a function of how separated the points in time [13] is and it is defined by

$$
R(\tau)=\lim _{T \rightarrow \infty} \frac{1}{T} \int_{0}^{T} F(u) F(u+\tau) \mathrm{d} u .
$$

$\tau$ is the correlation length or time interval. We show the autocorrelation plots depending on different $\beta$ and $\gamma$ parameters in Figure 7. The autocorrelation plots have the same periods with time series of the solutions of GNDES and TNDES $(F(u))$. For each $\beta$ and $\gamma$ parameters, the motions are periodic in time. When the nonlinearity increases, period of the oscillation gets smaller.

The relation between the oscillation frequency and autocorrelation is defined by power spectrum. The power spectrum is the Fourier transform of the autocorrelation function:

$$
S(\omega)=\int_{-\infty}^{\infty} R(\tau) e^{-2 \pi \omega \tau} \mathrm{d} \tau
$$




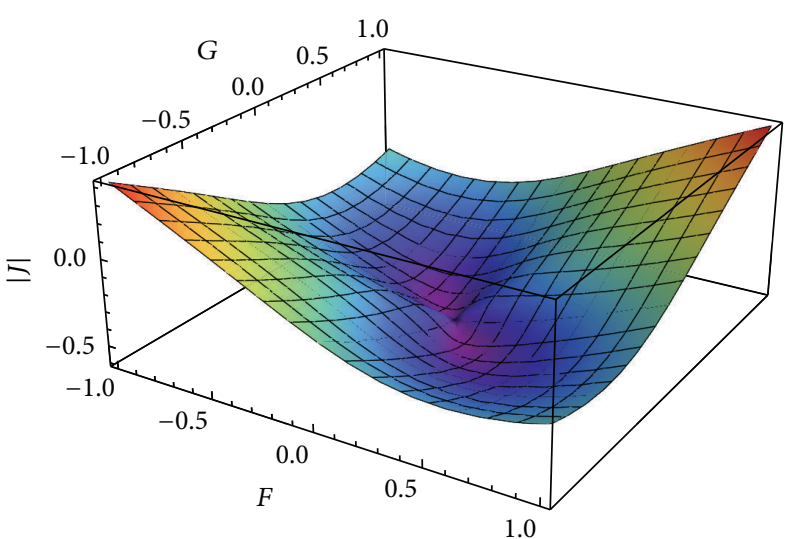

(a)

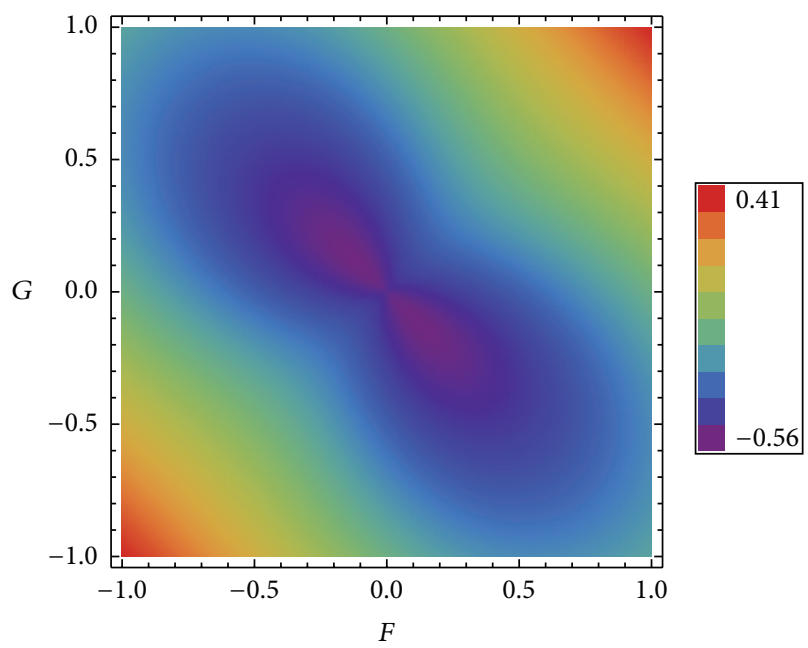

(b)

Figure 4: (a) 3D plot and (b) contour plot of $\operatorname{det}\left(J_{G}\right)$.

The power spectrum shows the energy distribution [13] over different frequencies of systems $(F(u))$. Power spectrum plots describe the periodic oscillation as the same as autocorrelation plots. However the solution $F(u)$ is almost sinusoidal but not exactly. Therefore, in power spectrums there is a fundamental frequency and there are some small harmonics close to the fundamental one. In Figure 8(a) we show the power spectrum of TNDES for various $\beta$ values. For $\beta=0.5$, $\omega_{0}=0.3777$ and its harmonics are 1.15 and 1.916 , for $\beta=1$, $\omega_{0}=0.7828$ and its harmonic is 2.376 , and finally for $\beta=1.5$, $\omega_{0}=1.21$. In Figure 8(b) we show the power spectrum of GNDES for various $\gamma$ values. For $\gamma=0.5, \omega_{0}=0.3462$ and its harmonics are 0.6952, 1.05, 1.399, and 1.751, for $\gamma=1$, $\omega_{0}=0.4719$ and its harmonic is 1.399 , and finally for $\gamma=1.5$, $\omega_{0}=0.7511$ and its harmonic is 2.262 .

\section{Conclusion}

In this paper we focus on the vector analysis and orientations of GNDES and TNDES to provide a better understanding of the behaviors of spinor-type instanton solutions of fermionic models in phase space for different dimensions and quantum spinor numbers. For this purpose the vector fields of fourdimensional Gursey instantons $\left(\alpha(A B)^{1 / 3}=\gamma=1\right)$ [4] and two-dimensional Thirring instantons $(\alpha(A B)=\beta=$ 1) [11] are constructed and it is shown that the systems are conservative along the flow. The situation is similar for the other $\beta$ and $\gamma$ values. In order to get more information on the orientation of systems in phase space, Jacobeans are calculated and plotted versus $F$ and $G$. This gives us an idea about whether the orientation of phase space area is preserved or not. The orientations of the four-dimensional Gursey instantons are similar to two-dimensional Thirring instantons in phase space.
Characterization of the fix points of systems are also briefly presented by calculating the characteristic eigenvalues. All eigenvalues are purely imaginary for both models so the fix points are elliptic except for $\beta=0$ and $\gamma=0$. For this parameter value, the fix points are hyperbolic as can be seen in Figures 1 and 3.

As additional information, we study systems using the hierarchical cluster analysis method. Similarities in $F(u)$ time series are displayed in Figures 5 and 6 by using the hierarchical cluster analysis method. As the result, we can say that the similarity of $F(u)$ data increases depending on the increment of the nonlinear control parameter for both models.

Finally we investigate autocorrelation and power spectrum of the systems with respect to the control parameters. It will be remarkable to notice that, as seen from Figure 7, the motions of systems are periodic for each value of the control parameters and as control parameters increase, periods of the oscillations get smaller and so frequency gets bigger for both models. Benefits from Fourier transform of the autocorrelation, fundamental frequency, and harmonics are calculated. As a result of our calculations we can say that the solution $F(u)$ is almost sinusoidal but not exactly the same. The systems have the same behaviors as the undamped harmonic oscillator.

In the view of obtained results, we can interestingly conclude that although models have different quantum spinor numbers and dimensions, the behaviors of four-dimensional spinor-type Gursey instantons are similar to those of twodimensional spinor-type Thirring instantons. This result is compatible with the knowledge of the critical behavior of a conformal symmetric quantum field theory near the fixed points [18-20]. Conformal symmetry fixes the 2-point and 3point correlation functions of primary fields since the only difference between two models is constants that depend on the interaction constant and dimensions and other details of 

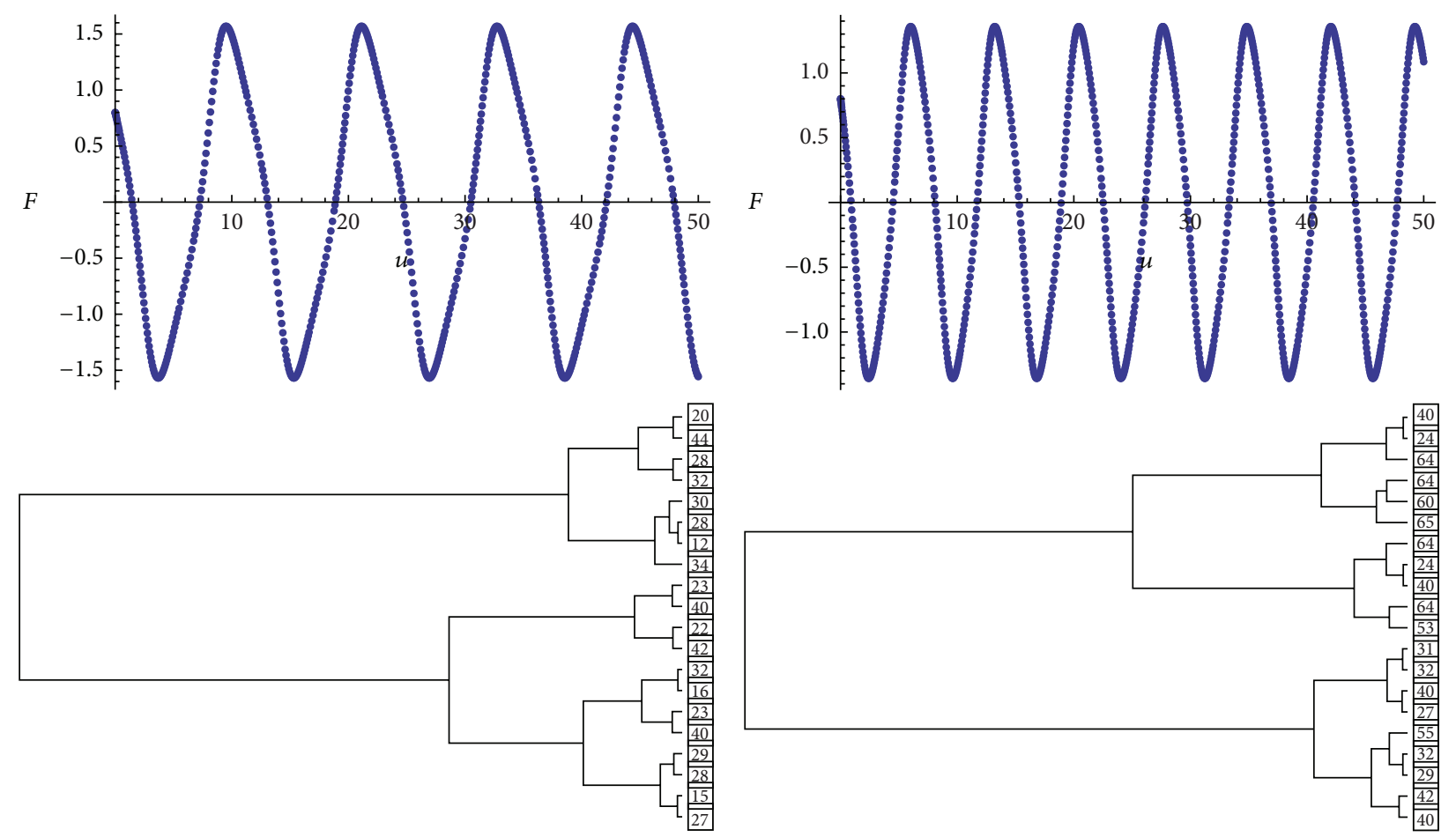

(a)

(b)
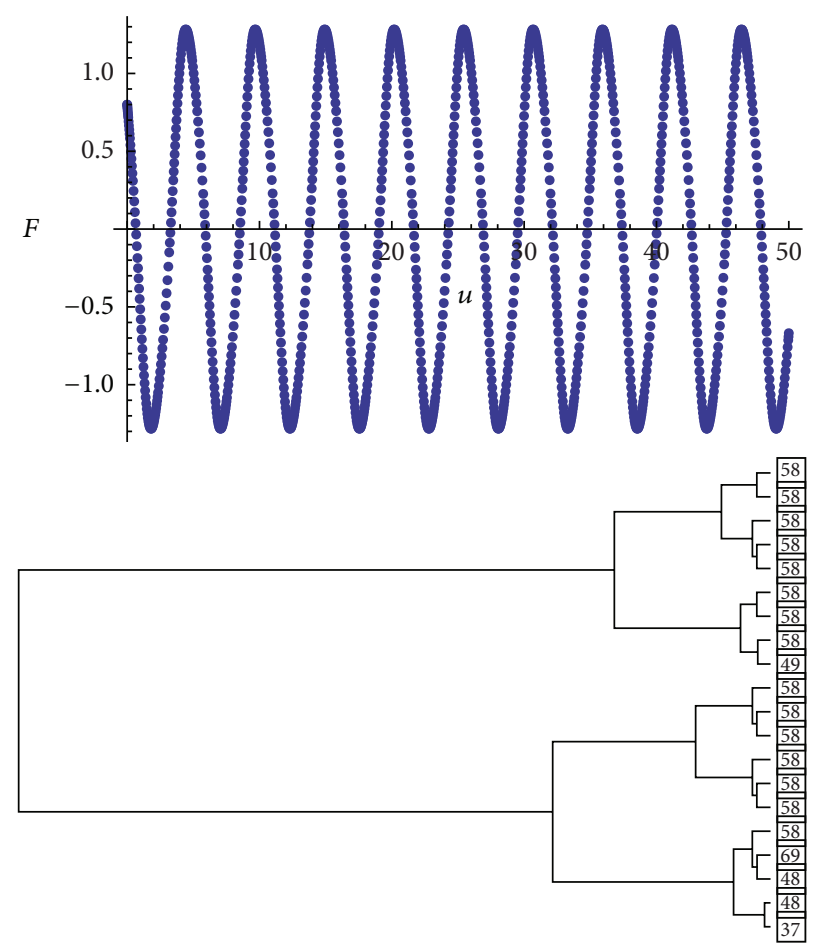

(c)

FIGURE 5: Time series and the resulting dendrogram from hierarchical clustering of TNDES for (a) $\beta=0.5$, (b) $\beta=1$, and (c) $\beta=1.5$. 

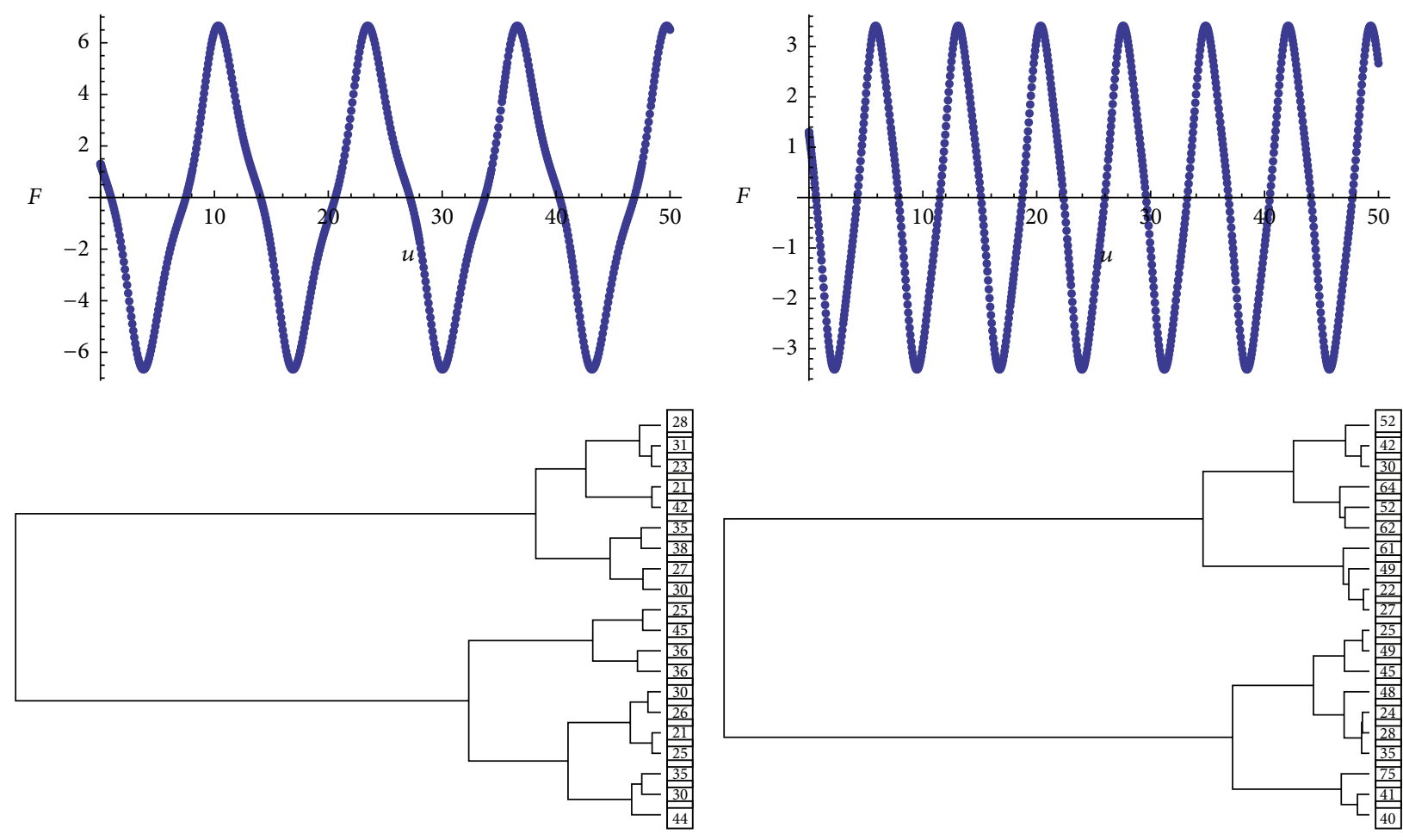

(a)

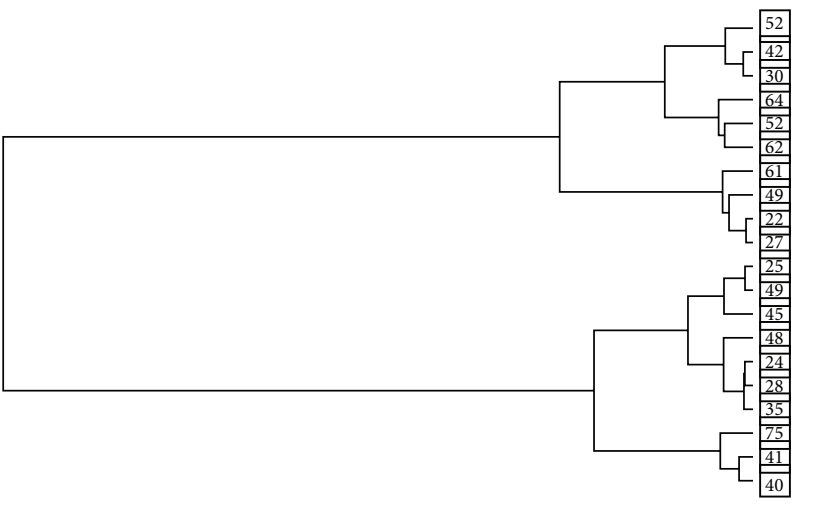

(b)
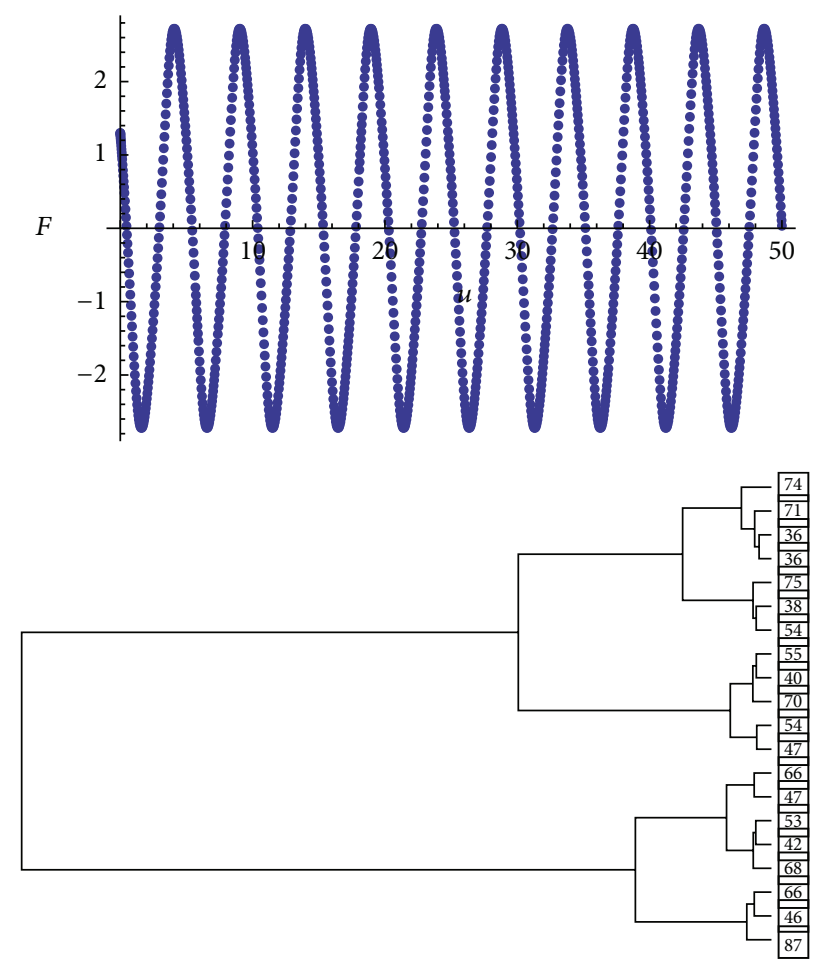

(c)

Figure 6: Time series and the resulting dendrogram from hierarchical clustering of GNDES for (a) $\gamma=0.5$, (b) $\gamma=1$, and (c) $\gamma=1.5$. 


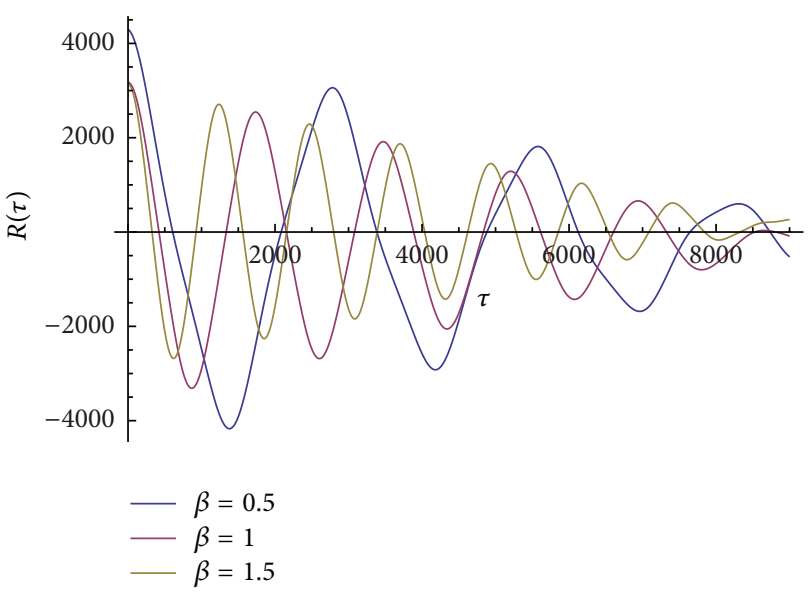

(a)

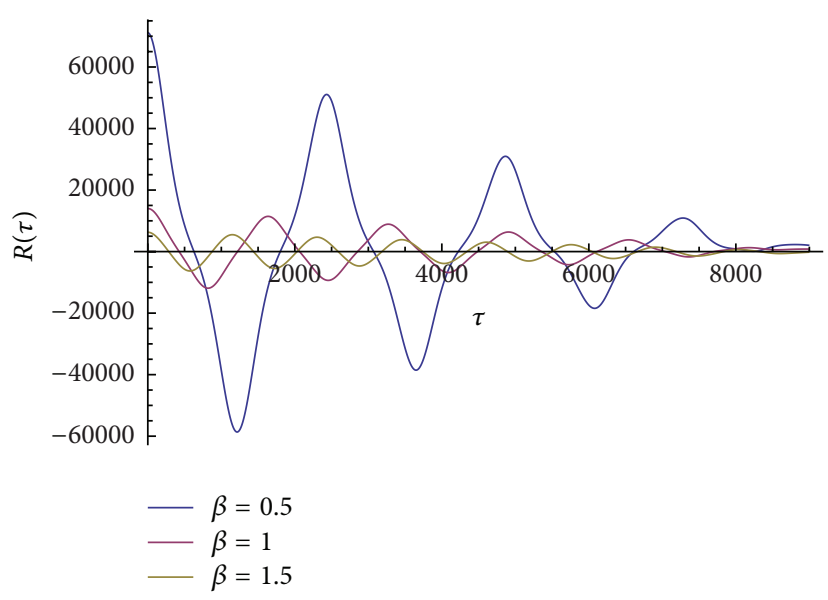

(b)

FIgURE 7: Autocorrelation displays of (a) TNDES and (b) GNDES.

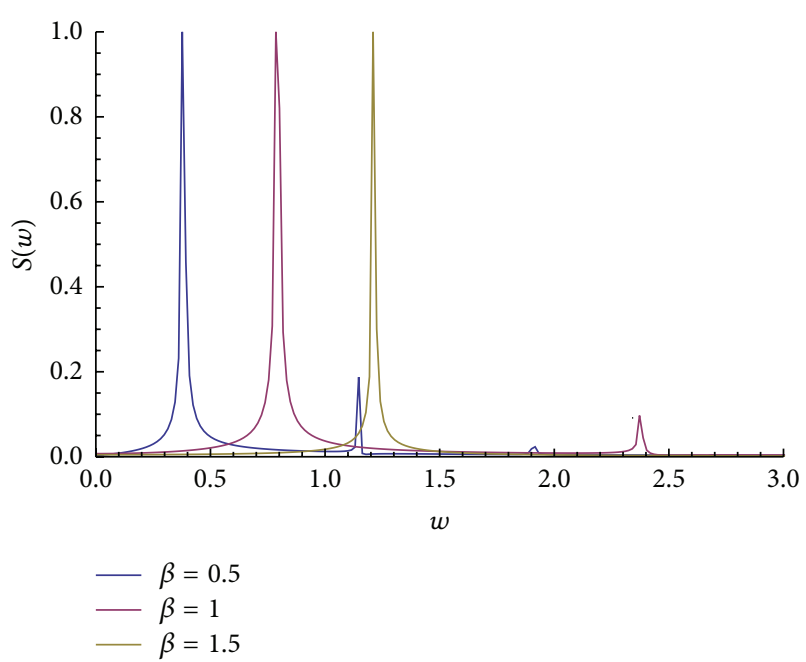

(a)

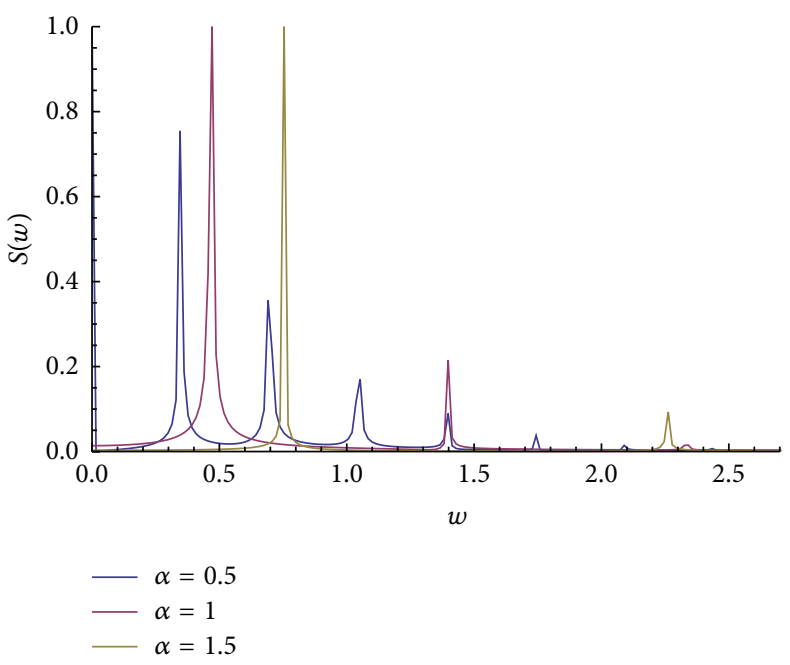

(b)

Figure 8: Power spectrum displays of (a) TNDES and (b) GNDES.

the models. Hence the classical solutions should have similar behaviors [18-24]. So we confirm that the behaviors of spinortype instantons in phase space are not dependent on the quantum fractional spinor number as well as dimensions $[8,9]$.

\section{Conflict of Interests}

The authors declare that there is no conflict of interests regarding the publication of this paper.

\section{Acknowledgments}

The authors would like to thank Professor Dr. K. Gediz Akdeniz and Professor Dr. Emine Rizaoglu for their support while preparing this paper. This work was supported by
Scientific Research Projects Coordination Unit of Istanbul University, Project nos. 33111 and 35737.

\section{References}

[1] W. Heisenberg, "Zur Quantentheorie nichtrenormierbarer Wellengleichungen," Zeitschrift für Naturforschung A, vol. 9, pp. 292-303, 1954

[2] F. Gursey, "On a conform-invariant spinor wave equation," Il Nuovo Cimento, vol. 3, no. 5, pp. 988-1006, 1956.

[3] F. Kortel, "On some solutions of Gursey's conformal-invariant spinor wave equation," vol. 4, pp. 210-215, 1956.

[4] K. G. Akdeniz, "On classical solutions of Gursey's conformalinvariant spinor model," Lettere al Nuovo Cimento, vol. 33, no. 2, pp. 40-44, 1982.

[5] K. G. Akdeniz, M. Arik, M. Durgut, M. Hortaçsu, S. Kaptanoğlu, and N. K. Pak, "The quantization of the Gürsey model," Physics Letters B, vol. 116, no. 1, pp. 34-36, 1982. 
[6] M. Hortacsu, B. C. Lutfuoglu, and F. Taskin, "Gauged system mimicking the Gürsey model," Modern Physics Letters A, vol. 22, pp. 2521-2532, 2007.

[7] M. Hortacsu and B. C. Lutfuoglu, "Renormalization group analysis of a Gursey model inspired field theory," Physical Review D, vol. 76, Article ID 105010, 2007.

[8] F. Aydogmus, The behavior of spinor type instanton attractors in phase space [Ph.D. thesis], Physics Department, Institute of Science, Istanbul University, 2012.

[9] F. Aydogmus, B. Canbaz, C. Onem, and K. G. Akdeniz, "The behaviours of Gursey instantons in phase space," Acta Physica Polonica B, vol. 44, no. 9, pp. 1837-1845, 2013.

[10] W. E. Thirring, "A soluble relativistic field theory," Annals of Physics, vol. 3, pp. 91-112, 1958.

[11] K. G. Akdeniz and A. Smailagić, "Classical solutions for fermionic models," Il Nuovo Cimento A, vol. 51, no. 3, pp. 345357, 1979.

[12] G. Benfatto and V. Mastropietro, Rigorous construction of the Thirring model: Ward-Takahashi Identities, Schwinger-Dyson Equations and New Anomalies, Universita Degli Studi di Roma "La Sapienza", 2004.

[13] J. M. T. Thompson and H. B. Stewart, Nonlinear Dynamics and Chaos, John Wiley \& Sons, New York, NY, USA, 2nd edition, 2002.

[14] X. Wang, K. Smith, and R. Hyndman, "Characteristic-based clustering for time series data," Data Mining and Knowledge Discovery, vol. 13, no. 3, pp. 335-364, 2006.

[15] B. Canbaz, C. Onem, F. Aydogmus, and K. G. Akdeniz, "From Heisenberg ansatz to attractor of Thirring Instanton," Chaos, Solitons \& Fractals, vol. 45, no. 2, pp. 188-191, 2012.

[16] F. Aydogmus, "Dynamics of excited instantons in the forced gursey nonlinear differential equations system," In press.

[17] D. K. Arrowsmith and C. M. Place, Dynamical Systems, Chapman and Hall Mathematics Series, Chapman \& Hall, London, UK, 1992.

[18] A. M. Polyakov, "Conformal symmetry of critical fluctuations," JETP Letters, vol. 12, no. 12, pp. 381-383, 1970.

[19] A. A. Migdal, "Conformal invariance and bootstrap," Physics Letters B, vol. 37, no. 4, pp. 386-388, 1971.

[20] G. Parisi and L. Peliti, "Calculation of critical indices," Lettere al Nuovo Cimento, vol. 2, no. 13, pp. 627-628, 1971.

[21] G. Mack and I. T. Todorov, Conformal Invariant Green Functions without Ultraviolet Divergences, ICTP, Trieste, Italy, 1971, preprint IC/71/139.

[22] I. T. Todorov, "Conformaly invariant quantum field theory," in Strong Interaction Physics, W. Ruhi and A. Vancura, Eds., vol. 17 of Lecture Notes in Physics, pp. 270-299, Springer, New York, NY, USA, 1973.

[23] G. Mack, "Conformal invariance and short distance behavior in quantum field theory," in Strong Interaction Physics, W. Ruhi and A. Vancura, Eds., vol. 17 of Lecture Notes in Physics, pp. 300-334, Springer, New York, NY, USA, 1973.

[24] A. F. Grillo, "Kinematical aspects of ccnformal invariance," in Strong Interaction Physics, W. Ruhi and A. Vancura, Eds., vol. 17 of Lecture Notes in Physics, pp. 335-342, Springer, New York, NY, USA, 1973. 

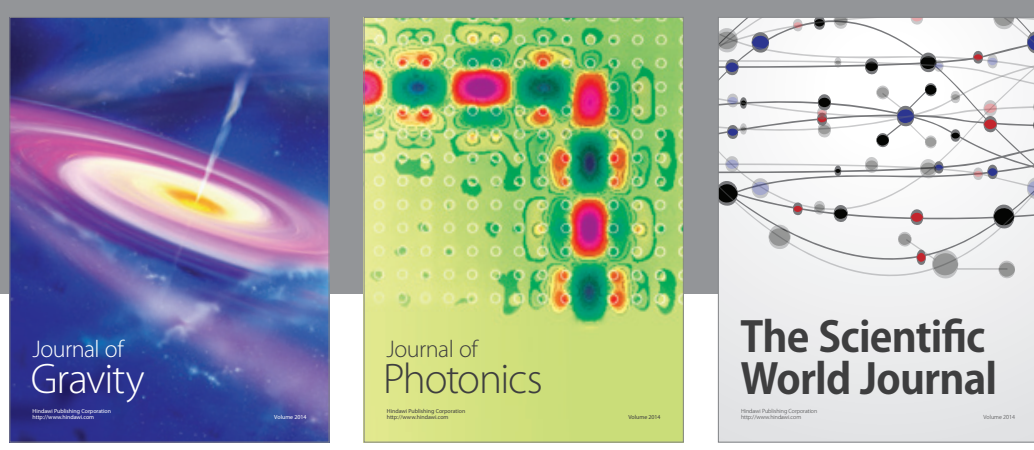

The Scientific World Journal
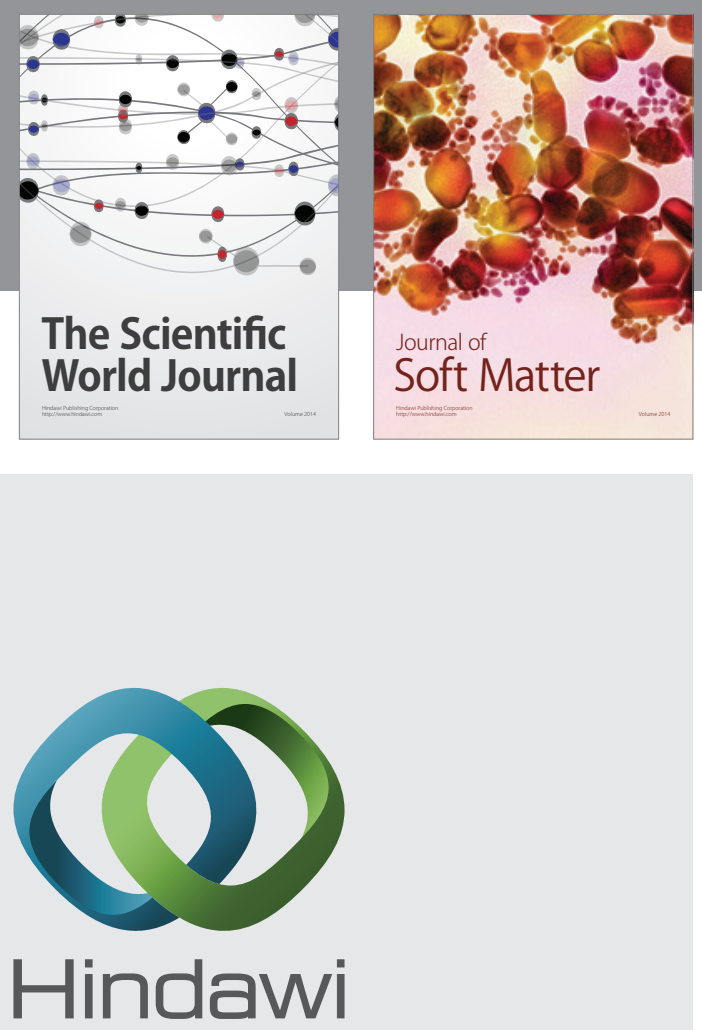

Submit your manuscripts at

http://www.hindawi.com

nternational Journal of

Statistical Mechanics
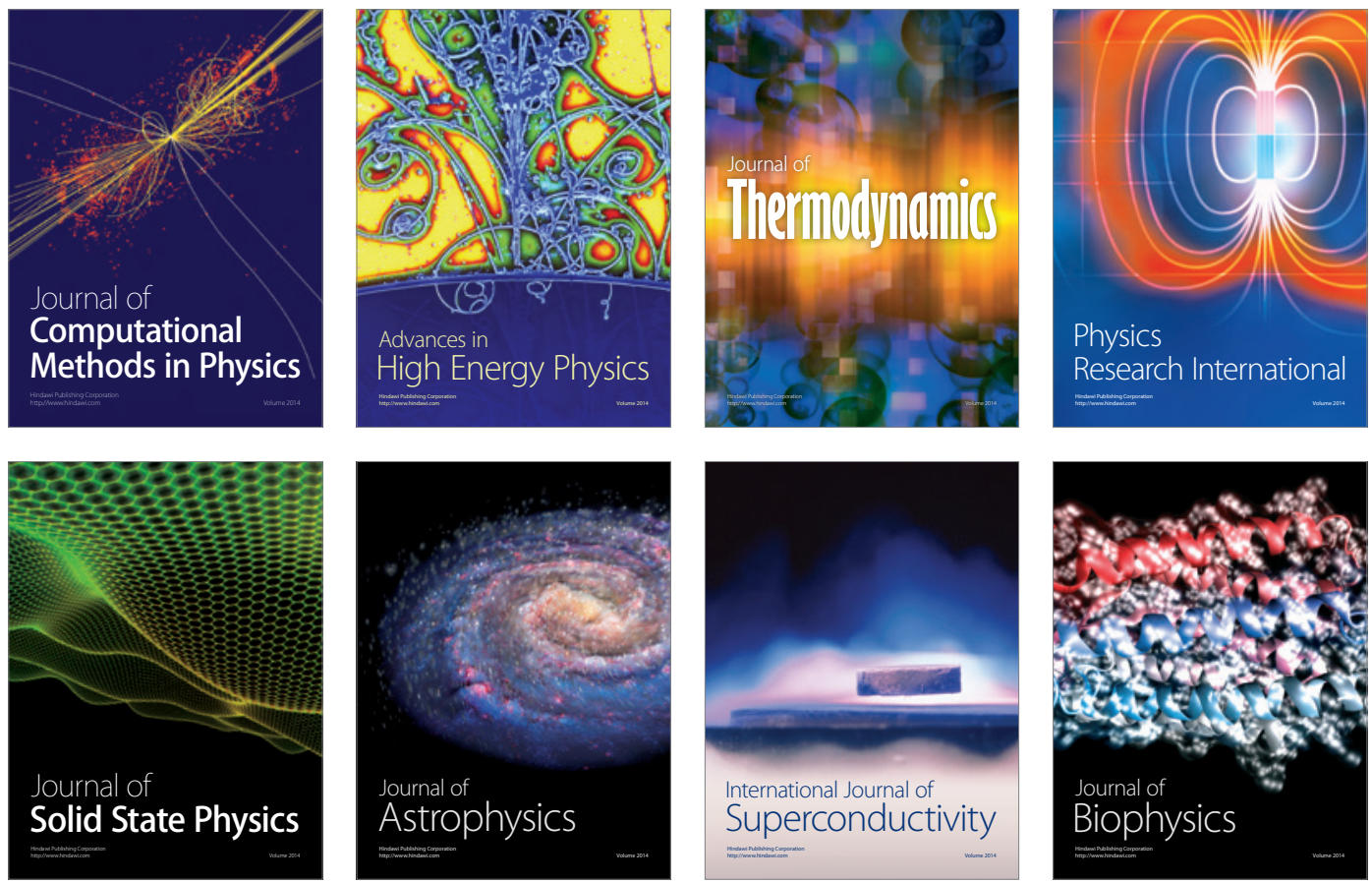
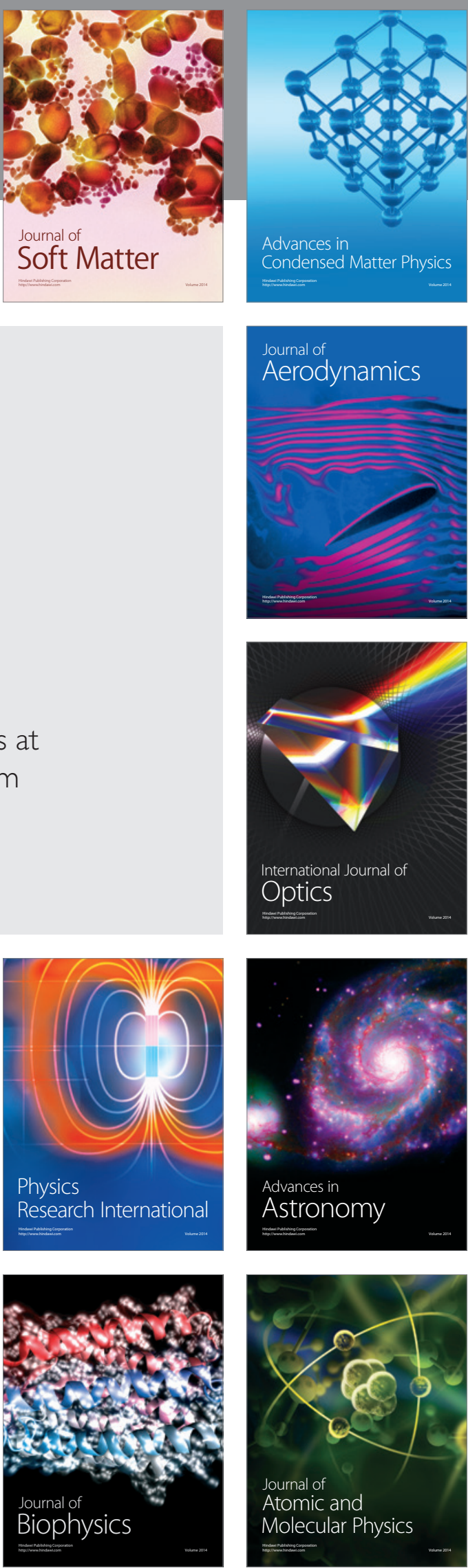